\title{
Maximum-Likelihood Principle for Determining Positions in Neutron Scintillation Detectors
}

\author{
J. Schelten, R. Engels, and R. Reinartz
}

\begin{abstract}
Pulse height division is generally used to determine a position channel for storing detection events in position-sensitive detectors (PSDs). An alternative method based on the maximum likelihood principle is discussed. This principle is modified in order that the storage of events can be done online. Analytic expressions for the spatial resolution are derived and evaluated Both detection methods are simulated for linear PSDs. Results on uniformity, linearity, stability and spatial resolution are presented. The major specific advantage of the proposed method is an improved uniformity of the response along the entire detector.
\end{abstract}

\section{INTRODUCTION}

$\mathbf{U}$ SING position-sensitive detectors (PSDs) with a large sensitive area, high resolution, and linear response is frequently the only way to improve the experimental situation in nuclear medicine and nuclear physics.

In positron emission tomography (PET), the total amount of detectable coincident gammas can not be increased because the dose of radioactive pharmaceuticals a patient can carry, and the data acquisition time a patient can tolerate, are already at their upper limits. Thus, only the detection of all emitted gamma rays can improve PET images.

In neutron scattering experiments, the scattering data often have large error bars. A drastic increase of neutron flux at the sample cannot be expected from advanced neutron sources, such as future high-flux reactors or pulsed spallation neutron facilities. Only with detectors which detect all scattered neutrons is the situation is improvable.

These two examples demonstrate that progress in positionsensitive detection of high energy gammas and thermal neutrons plays a key role.

In scintillation detectors equipped with phase modulation (PM) arrays [1], [2], or with position-sensitive PMs [3], [4], in semiconductor strip diodes [5], and in microstrip gas detectors [6], [7] usually, the position channel for storing a detection event is determined by pulse height division. This method is certainly not the best although, in many cases, excellent results have been obtained.

For high count rates, it is not tolerable that all detector signals are used to determine a position estimate because in this case a completely wrong answer is obtained if two or more events occur at the same time. The alternative would be to obtain position information only from those few PM signals which con-

Manuscript received February 28, 2000; revised June 15, 2000 and September 6, 2000.

The authors are with the Institut für Schicht- und Ionentechnik and Zentrallabor für Elektronik, Forschungszentrum Jülich GmbH, D-52425 Juelich, Germany.

Publisher Item Identifier S 0018-9499(00)10279-5. tribute mostly to the position determination, because this allows to treat simultaneously occuring events correctly. For detectors which operate at pulsed neutron sources, where bursts of neutrons need to be treated, this argument plays an important role. In this context, the maximum-likelihood principle is helpful.

In the fundamental paper by Tanaka [8], the position functions of scintillation detectors was investigated in general. The position function has the PM signals as argument and yields an estimate of the event position. Tanaka derived relations of the position function for getting best resolution and linear response, and suggested how to realize the condition with a delay line readout electronic. His ideas were experimentally verified in [9] and [10]. Five years later, Gray and Macovski [11] were the first to discuss the position determination with the maximum-likelihood (ML) principle. There, it was shown that the ML estimate implies a solution that is remarkably similar to the "optimum position arithmetic" as derived by Tanaka. As a consequence, the method implies not only a linear detector response but also a spatial resolution which is close to its optimum value. In the following years attempts were made to implement the ML principle in scintillation detectors with analog electronic [12]-[14]. In addition digital implementation was realized for large scintillation detectors consisting of small modules with only four PMs. For the module signals the ML method was applied successfully to determine the storage locations via lookup tables. [15] and [16]. The lookup tables were filled with most-probable event positions which are calculated from two PM row and column sum signals.

In our laboratory, the detector development focuses on linear and area scintillation detectors for thermal neutrons. The new detector electronic consists of free-running analog-to-digital converters (ADCs) and fast digital data treatment in field-programmable gate arrays (FPGAs) and digital signal processors (DSPs). In these detectors, simultaneously occuring events must be recorded, for which the ML method is helpful, as was mentioned above.

Thus far, simulations have been done for the one-dimensional detector, in which only the two most significant PM signals are selected for position determination and for the two-dimensional case where four PMs are needed to determine the $x$ and $y$ positions.

The simulations clearly demonstrated that the separation of variables, as discussed in [15], does not yield satisfactory results. i.e., the two-dimensional case does not separate well into two linear cases in which the $x$ coordinates are determined from the sum signals of columns and the $y$ coordinate from sum signals of rows. For the two-dimensional detector, a modification of the ML method has to be developed which yields a vector 
position from the four most significant PMs in an efficient way which can be applied to online data with $1 \mathrm{MHz}$ repetition rate in DSP.

Because of the large difference in the concepts for the linear and the area detector, in this paper only the linear case is presented. For the area detector, it must be shown first that the required data calculations to obtain the maximum probability, can be done fast enough in DSPs.

In Section II, the concept is described. There, the exact ML treatment is modified such that an online event storage is possible. Analytical expressions for the spatial resolution are derived.

In Sections III and IV, more practical results are presented. They are obtained by simulating linear scintillation PSDs with five PMs. The simulations allow a comparison of the ML method with the classical center of mass method.

Finally, in Section V, the results are summarized and the value of both methods is discussed critically.

\section{MAXIMUM-LIKELIHOOD PRINCIPLE}

\section{If \\ $S_{i}$ \\ $B_{i}(x)$}

$N_{c}$

$n$

$x_{k}$

$P\left(S_{i}, B_{i}(x)\right)$ is the probability of the Poisson. Then, the most probable event position is defined in

$$
x_{E}=\left\{x_{k}: \operatorname{Max}\left[\prod_{i}^{n-1} P\left(S_{i},\left\langle S_{i}\left(x_{k}\right)\right\rangle\right) ; 0 \leq k \leq N_{c}\right]\right\} .
$$

This equation was discussed almost 25 years ago by Gray and Macovscy (1976) [11].

With sufficient accuracy, the probability functions can be approximated by Gaussian distributions as done in (2)

$$
P_{(S i, B i(x))}=\frac{1}{\sqrt{2 \pi B_{i}(x)}} \exp \frac{-\left(S_{i}-B_{i}(x)\right)^{2}}{2 B_{i}(x)} .
$$

This approximation and the next one, which leads to equation (4), are necessary to make if one is forced to find the position of maximum probability by an on-line calculation within a very short time before the next event occurs.

The probability functions are normalized to one according to

$$
\int P\left(S_{i}, B_{i}(x)\right) d S_{i}=1
$$

The valuable feature of the maximum-likelihood principle is that the expectation value of the position event is identical with the true event, i.e., $\left\langle x_{E}\right\rangle=x$. Consequently, the detector response is exactly linear.
Since the probability functions in (2) are sharply peaked, the maximum-likelihood principle is well approximated by the least square deviation principle

$$
x_{E}=\left\{x_{k}: \operatorname{Min}_{\gamma}\left[\sum_{i=0}^{n-1} \frac{\left(\gamma S_{i}-B_{i}\left(x_{k}\right)\right)^{2}}{2 B_{i}\left(x_{k}\right)}\right] 0 \leq k \leq N_{c}\right\} .
$$

The parameter $\gamma$ has been introduced because the minimum search result should not depend on the absolute value of the signals $S_{i}$, but solely on their ratios.

Reducing the minimum calculation to only those two signals $S_{i-1}$ and $S_{i}$, which are the largest neighbored pairs among all $n-1$ possible pairs, the minimization with respect to $\gamma$ leads to

$$
\begin{array}{r}
x_{E}=\left\{x_{k}: \operatorname{Min}\left[\frac{\left(S_{i} B_{i-1}\left(x_{k}\right)-S_{i-1} B_{i}\left(x_{k}\right)\right)^{2}}{S_{i}^{2} B_{i-1}\left(x_{k}\right)+S_{i-1}^{2} B_{i}\left(x_{k}\right)}\right]\right. \\
\left.\cdot 0 \leq k \leq N_{c}\right\} .
\end{array}
$$

Obviously, the minimum search for two signal pairs $\left(S_{i-1}, S_{i}\right)$ and $\left(\lambda S_{i-1}, \lambda S_{i}\right)$ yields the same event position for all values of $\lambda$.

This simplification is deliberately done because lookup tables can then be used. If more than two PM signals are involved in the position estimate, the lookup table becomes too large in size. It is the purpose of this treatment to investigate how much impact this simplification has on the spatial resolution.

With the linear relation of channel positions $x_{k}$ and channel number $k$

$$
x_{k}=L_{D} \frac{k}{N_{c}-1} 0 \leq k \leq N_{c}-1
$$

one determines a minimum channel number according to (7)

$$
k_{\min }=\left\{k: \operatorname{Min}\left[\frac{\left(S_{i} B_{i-1}\left(x_{k}\right)-S_{i-1} B_{i}\left(x_{k}\right)\right)^{2}}{S_{i}^{2} B_{i-1}\left(x_{k}\right)+S_{i-1}^{2} B_{i}\left(x_{k}\right)}\right]\right\} .
$$

The minimum search can be done offline for all possible detector signals, and the results can be tabulated for later use.

The table has three integer entries, $\left[i, S_{i-1}\right.$, and $\left.S_{i}\right]$. With an 8-bit ADC, the $S_{i-1}$ and $S_{i}$ both have 256 possible values. For a big detector with, e.g., $64 \mathrm{PMs}$, the number of entries is $64 \times 256 \times 256=410^{6}$. The content of the table is the calculated integer $k_{\min }$ from (7), i.e.,

$$
\operatorname{Tab}\left[i, S_{i-1}, S_{i}\right]=k_{\min } .
$$

The computer time to fill the table is moderate, about $10 \mathrm{~min}$ with a PC of $300 \mathrm{MHz}$.

For the simulations, a PM array of five PMs was sufficient to demonstrate all effects, and a signal of seven bits was accurate enough.

In this case, the table has a size $6.610^{4}$ entries and filling the table took about $10 \mathrm{~s}$.

Such prepared tables are used to handle a detector event. The event is represented by a set of signals $\left\{S_{0}, S_{1}, S_{2}, \ldots, S_{n-1}\right\}$ from which the pair of strongest signals is selected. The selection leads to the triplet $\left[i, S_{i-1}, S_{i}\right]$ and, via the tabulated 
values (8), to the $k_{\min }$ channel number. Finally, the content of this channel is incremented by one.

The spatial detector resolution is determined by the fluctuation of the statistical variables $S_{i-1}, S_{i}$, with variances

$$
\left\langle\left(S_{i}-\left\langle S_{i}\right\rangle\right)^{2}\right\rangle=\left\langle\left(S_{i}-B_{i}(x)\right)^{2}\right\rangle=B_{i}(x) .
$$

The variation of the minimum condition of (5) requires

$$
\partial\left[S_{i} B_{i-1}(x)-S_{i-1} B_{i}(x)\right]=0
$$

and leads to the following expression for the variance of point spread function:

$$
\begin{aligned}
& \sqrt{\left\langle(\delta x)^{2}\right\rangle} \\
& =\frac{1}{\sqrt{N_{t o t}}} \frac{\sqrt{1+B_{i}(x) / B_{i-1}(x)+B_{i-1}(x) / B_{i}(x)+1}}{\left|\frac{B_{i}(x)^{\prime}}{B_{i}(x)}-\frac{B_{i-1}(x)^{\prime}}{B_{i-1}(x)}\right|} .
\end{aligned}
$$

The variance is a function of the involved expectation functions $B_{i}(x)$ and their derivatives $B_{i}(x)^{\prime}$. In addition, it depends directly on the averaged total number $N_{\text {tot }}$ of involved electrons per event. This number is proportional to the sum $B_{i}(x)+$ $B_{i-1}(x)$.

There must exist an expectation function $B_{i}(x)$ which minimizes the spatial resolution. For a homogeneous detector, the two functions $B_{i-1}(x)$ and $B_{i}(x)$ are not independent, since the relation $B_{i-1}(x)=B_{i}(x+a)$ then holds, where $a$ is the distance between adjacent PMs. Thus, the variational problem is reduced to only one function. Nevertheless, the problem remains difficult and the authors were not able to solve it.

\section{Pulse Height Division Method}

This classical estimate of event positions is based solely on the set of the statistical variables $\left\{S_{0}, S_{1}, S_{2}, \ldots, S_{n-1}\right\}$, and does not need information of the expectation functions. The event position is determined in a center of mass equation

$$
x_{E}=L_{D} \frac{\sum_{i=0}^{n-1} \alpha_{i} S_{i}}{\sum_{i=0}^{n-1} S_{i}}, \quad \alpha_{i}=\frac{i}{n-1} .
$$

In a first-order approximation the expectation value of $x_{E}$ is:

$$
\left\langle x_{E}\right\rangle=L_{D} \frac{\sum_{i=0}^{n-1} \alpha_{i} B(x)_{i}}{\sum_{i=0}^{n-1} B(x)_{i}}, \quad \alpha_{i}=\frac{i}{n-1} .
$$

This relation $\left\langle x_{E}\right\rangle$ versus the true position $x$ is a nonlinear function, and nonlinearities are particularly severe at the two detector ends.

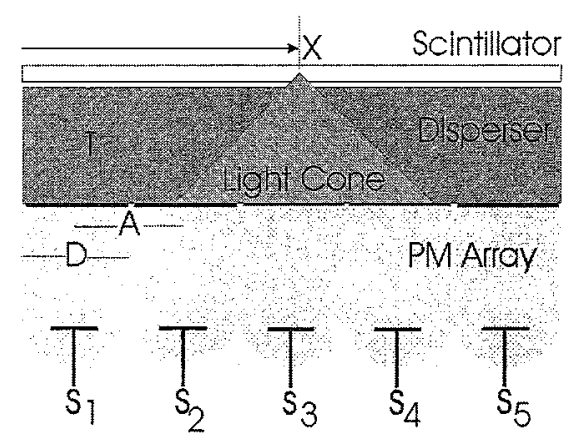

Fig. 1. Sketch of a linear scintillation detector with five PMs, a disperser, a scintillator, and an air gap.

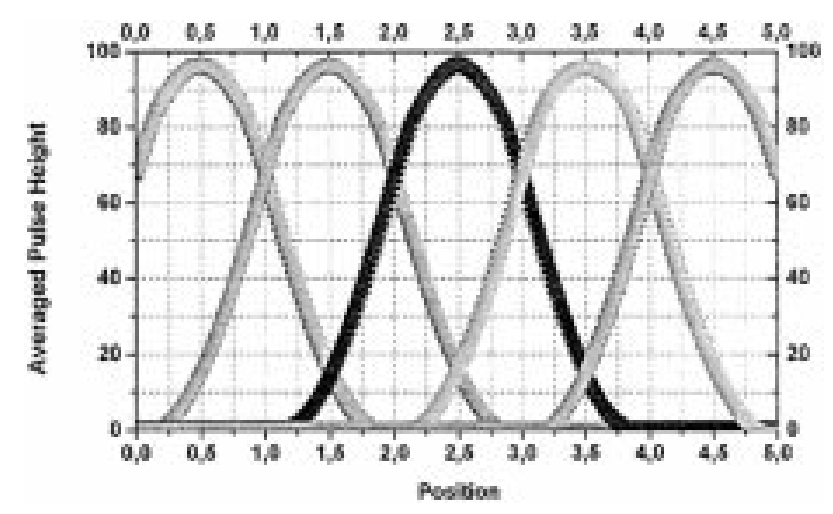

Fig. 2. Expectation functions $B_{i}(x)$ versus detector position for an array of five PMs with the reference parameters, given in the text.

The variance of point spread function which is a measure of spatial resolution is given in the following equation:

$$
\sqrt{(\delta x)^{2}}=\frac{\sqrt{\left\langle\left(x_{E}-\left\langle x_{E}\right\rangle\right)^{2}\right\rangle}}{\frac{d}{d x}\left\langle x_{E}\right\rangle} .
$$

The calculation utilizing the fluctuation variances of the signal $S_{i}$ leads to the following expression

$$
\sqrt{\left\langle(\delta x)^{2}\right\rangle}=\frac{1}{\sqrt{N_{t o t}}} \frac{\sqrt{\overline{(\alpha-\bar{\alpha}(x))^{2}}}}{\frac{d \bar{\alpha}(x)}{d x}} .
$$

The bar operations are explicitly expressed in the following two equations:

$$
\begin{gathered}
\bar{\alpha}(x)=\frac{\sum_{i=0}^{n-1} \alpha_{i} B_{i}(x)}{\sum_{i=0}^{n-1} B_{i}(x)} \\
\overline{(\alpha-\bar{\alpha}(x))^{2}}=\frac{\sum_{i=0}^{n-1}\left(\alpha_{i}-\bar{\alpha}(x)\right)^{2} B_{i}(x)}{\sum_{i=0}^{n-1} B_{i}(x)} .
\end{gathered}
$$

Again, the variance of a point spread function is determined by two factors, the averaged total number $N_{t o t}$ of involved elec- 


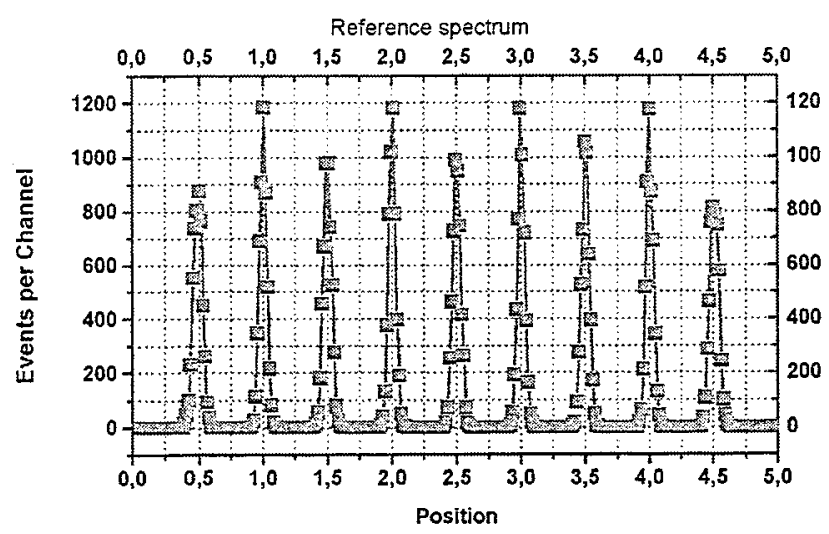

(a)

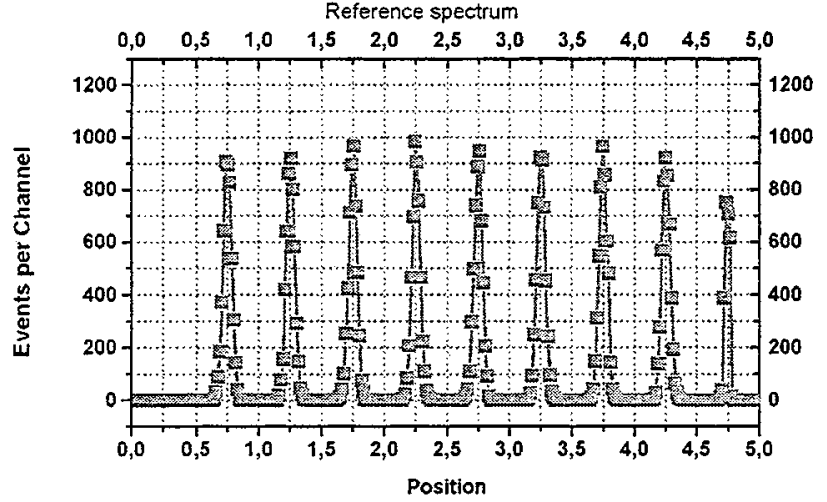

(b)

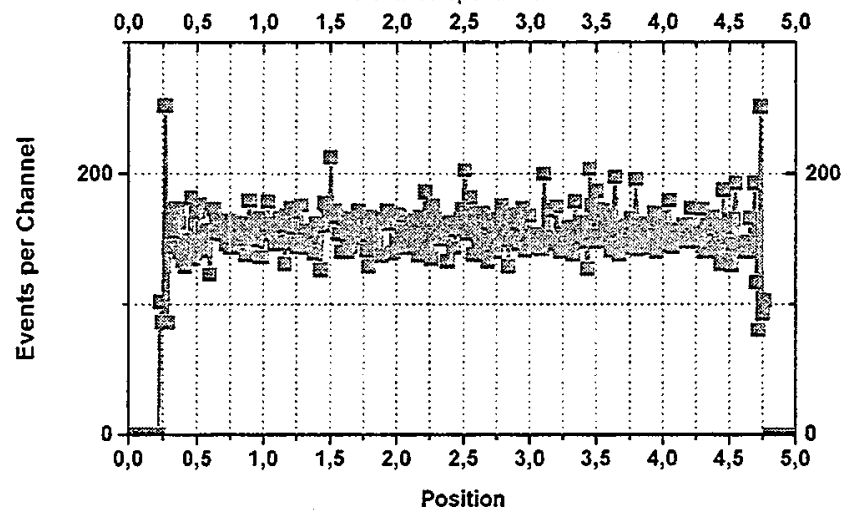

(c)

Fig. 3. (a) and (b) Point spread functions generated at nine detector positions with data handling due to the maximun-likelihood principle. (c) Intensity versus position channel accumulated at hundreds of hundreds of homogeneously distributed detector positions with data handling due to the maxium-likelihood principle. This plot simulates homogeneous illumination of a detector.

trons generated at the photo cathodes per event and the expectation functions $B_{i}(x)$ with their derivatives. Both contributions cause a position dependence of the resolution.

Again, it is a major unsolved task to find the expectation function $B_{i}(x)$ which minimizes the spatial resolution, and then try to realize the optimal distributions.

\section{Simulation Results}

The simulation of both detection methods was done for a linear scintillation detector with five PMs.

As sketched in Fig. 1, the generated light from an absorbed neutron or gamma ray in the scintillator, is distributed on the PM photo cathodes. Because of an air gap between scintillator and disperser, light rays outside the indicated cone are totally reflected at the scintillator faces and cannot reach the photo cathodes.

The light distribution can be influenced by two parameters: 1) with the cone angle $2 \alpha$ and 2) with the disperser thickness $T$. Reference values for the detector simulations are distance $A=1$ and diameter $D=1$ of the PMs, cone angle $\alpha=45^{\circ}$ and disperser thickness $T=0.9$. In addition, the total number of generated photo electrons is an adjustable parameter. The reference value is 400 . With these reference values the simulated detector approaches the JULIOS detector [1], [3], which is used in neutron powder diffractometers at various reactor stations.
The distributions as measured with the JULIOS detector were well represented by such distributions as shown in Fig. 2. Due to the air gap between disperser and PM, the distributions do not show the long tails as generally observed in Anger cameras. The curves are little effected by PM nonuniformities because the signals are resulting from averaging integrals over the whole photo-cathode area.

In Fig. 2, the five expectation functions $B_{i}(x)$ are shown versus the detector position. They have been calculated for the reference detector assuming a uniform PM sensitivity. Experimentally, these curves can be determined by scanning a narrow beam across the detector and by determining the pulse height average. With these distributions, the table $\operatorname{Tab}\left(i, S_{i-1}, S_{i}\right)$ is filled with calculated $k_{\text {min }}$ channels for all possible values $0<=$ $S_{i-1}, S_{i}<=127$, and $0<=i<=4$. This table is then used for simulating measurements.

The steps for handling a detector event at a given position due to the maximum-likelihood method are as follows.

1) For the given position, a set of $\left\{B_{1}(x), B_{2}(x), \ldots\right.$, $\left.B_{n-1}(x)\right\}$ values exist, which have the meaning of PM signals on average for this position. With a Poisson random number generator a set of signals $\left\{S_{1}, S_{2}\right.$, $\left.\ldots, S_{n-1}\right\}$ is obtained. Such a set represents a detector event.

2) From this set of signals, the largest signal pair $\left(S_{i-1}, S_{i}\right)$ is selected. 


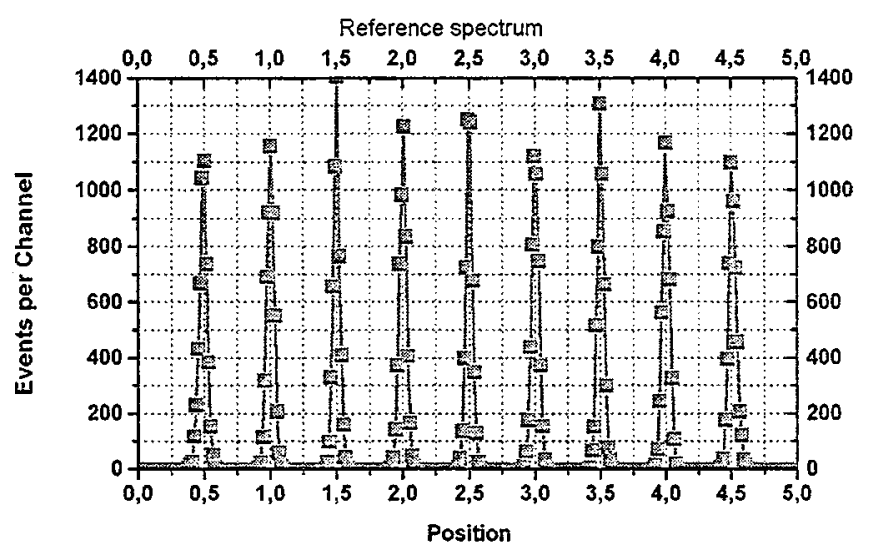

(a)

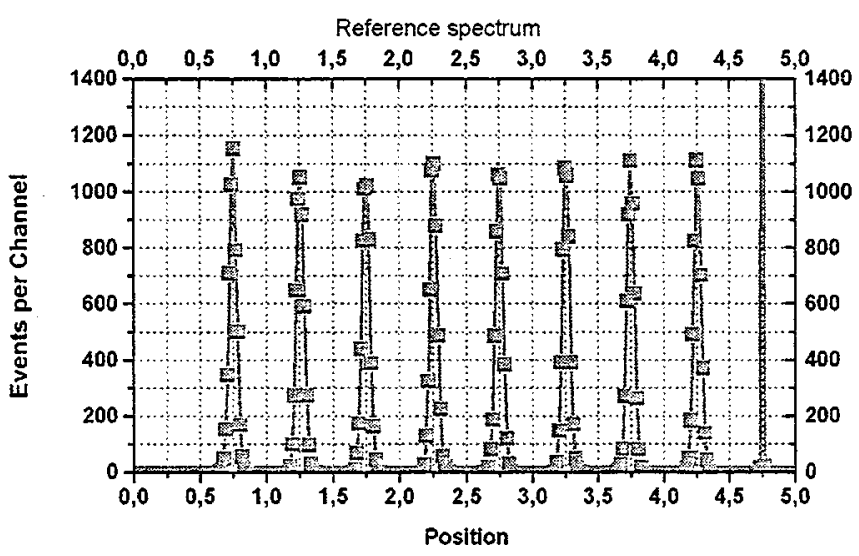

(b)

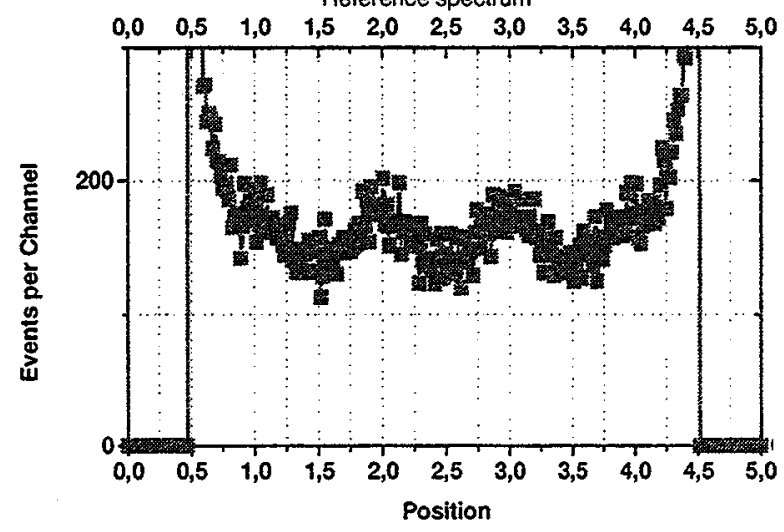

(c)

Fig. 4. (a) and (b) Point spread functions generated at nine detector positions with data storage due to the center of mass method. (c) Intensity versus position channel accumulated at hundreds of homogeneously distributed detector positions with data storage due to the maxium-likelihood principle. This plot simulates homogeneous illumination of a detector.

3) This signal pair is used as entry of the table in order to get the most probable channel. The content of this channel is incremented by one.

In Fig. 3(a) and (b), point-spread functions are generated by creating events at nine detector positions. The FWHM values of the generated peaks are position dependent. If one assumes 400 electrons per event on average, the FWHM is about $10 \%$ of the number of channels per PM (64 channels per PM) or $10 \%$ of the PM distance.

For a homogeneous generation of detector events along the detector, one gets a constant intensity profile. The channel contents fluctuate only randomly about an average content, see Fig. 3(c). No peculiarities are observed at the middle between two consecutive PMs nor at the center of a PM.

The results are compared with simulated data based on the center of mass principle. The steps for handling a detector event are similar.

1) For the given position, a set of $\left\{B_{1}(x), B_{2}(x), \ldots\right.$, $B_{n 1}(x)$ \} values exist, which have the meaning of PM signals on average for this position. With a Poisson random number generator a set of signals $\left\{S_{1}, S_{2}, \ldots, S_{n-1}\right\}$ is obtained which represents the detector event.

2) From this set, the ratio due to (11) is evaluated.

3) The result is a channel number. The content of this channel is then incremented by one.
In Fig. 4(a) and (b), two-point spread functions with nine peaks are shown. The peaks have position dependent FWHM values. On an average the peak widths are somewhat smaller than in Fig. 3(a) and (b).

For a homogeneous generation of detector events along the detector, one gets an oscillating intensity profile with strong wings on both detector ends. This shape is caused by the nonlinearity of the detector response, see Fig. 4(c).

In Fig. 5, the analytic expressions of the spatial resolution are numerically evaluated and plotted versus the detector position for the two cases of maximum-likelihood principle and pulse height division method. The variances are converted to FWHM in units of channels and plotted versus the detector position. The calculated values are in good agreement with the widths of the peaks in Fig. 3(a) and (b) and Fig. 4(a) and (b).

In Fig. 6, the integral linearity is investigated. For many detector events, intensity peaks are generated using both methods. The centers of these peaks are plotted versus the detector position. One observes the exact linear relation in the maximum-likelihood case and the nonlinear behavior in the center of mass case. The nonlinearity is present everywhere and strongest at the two detector ends.

The data of Fig. 7 were generated by deliberately varying the averaged total number of electrons per event, e.g., from 800 to 25 corresponding to an excellent to a very poor scintillator, 


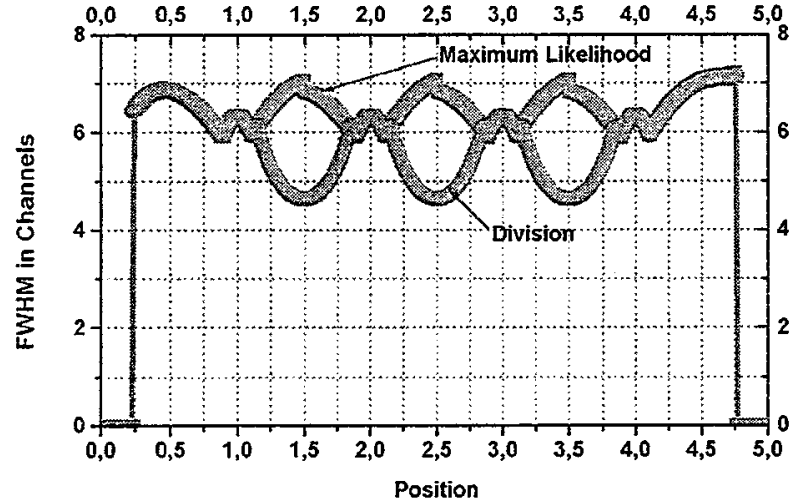

Fig. 5. FWHM in units of position channels versus detector position as calculated from analytical resolution expressions (10) and (14) for both methods (FWHM $=2.35\left(N_{P M} / A\right)\left\langle(\delta x)^{2}\right\rangle^{1 / 2}$; with $N_{P M}=64$ Channels per PM and $A=1$ of the PM distance.

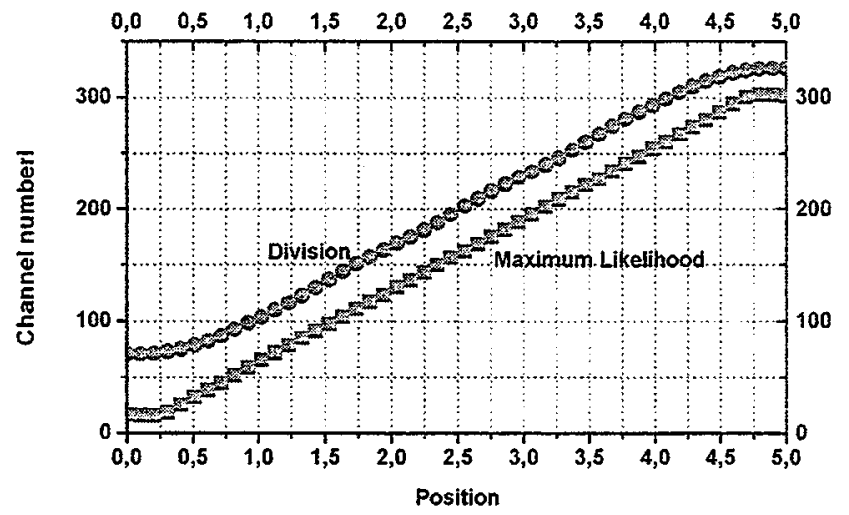

Fig. 6. Peak position channel versus detector position as determined by generating 100 events at 50 detector positions with both methods.

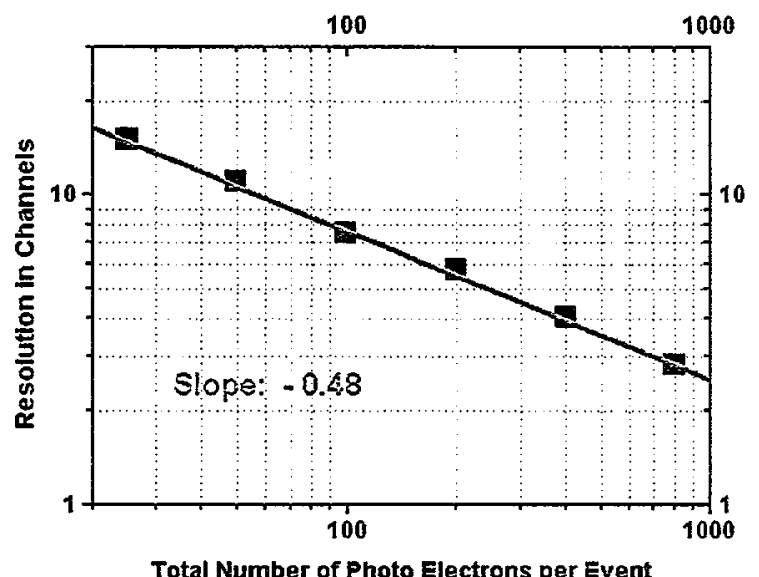

Fig. 7. FWHM as determined from point spread functions versus the total number electrons per event in a double logarithmic scale.

respectively. From generated peaks, the FWHM was determined and plotted versus the electron number in a double logarithmic scale. The $N^{-1 / 2}$ behavior, as described in (10), is verified by getting a least squares linear fit with a slope of 0.48.

In this simulation experiment, the expectation functions were deliberately varied by increasing the disperser thickness from 0.8 to 1.5 times the PM distance. Simultaneously, the averaged number of electrons per event was adjusted such that the sum of

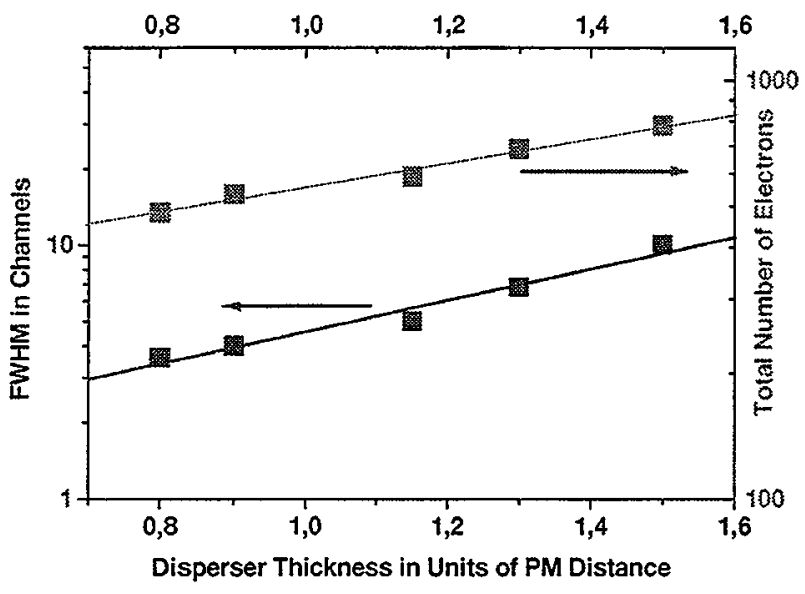

Fig. 8. FWHM as determined from point spread functions versus the extension of the expectation functions at the PM cathode plane in units of PM distance. The total number of electrons is adjusted such that the number of electrons of both involved signals is always 400 on average. The adjusted total number of electrons is also plotted in this plot.

the two signals which are used to determine the channel position via the table is always 400 on average. The plots of Fig. 8 indicate that the shape of the expectation function has a strong influence on the FWHM on the width of the point spread function since there is an increase by a factor of three. If one would not correct for the averaged number of electrons for the signal pair, the FWHM would become even broader with increasing disperser thickness (again by a factor of about three).

In further simulation experiments, the height or the width of one of the expectation functions $B_{i}(x)$ was changed deliberately by $10 \%$, and the effect on detector response was determined. In general, in the intensity distribution for homogeneous illumination a $10 \%$ change causes not more than $10 \%$ fluctuations, while the influence on resolution is even less. This indicates that the detector response is moderate and smooth.

\section{Summary AND Discussion}

The characteristic features of both methods are summarized in the Table I.

The strength of the center of mass method is that its application needs no more information than the signals themselves. The spatial resolution has been found to be slightly higher in all investigated cases. Nevertheless, this is not a proven rule for all expectation functions.

The strength of the maximum-likelihood method is the perfect linearity, and that one gets satisfactory results by involving only two PM signals. This limitation has, for semiconductor detectors, the enormous advantage that the noise of all other signals cannot diminish the spatial resolution. Furthermore, this method does not ask for a precise adjustment of the average signal heights.

The weakness of the center of mass method is its nonlinearity, which is moderate except at the detector ends, where it is strong.

The weakness of the maximum-likelihood method is certainly the need to provide a complete set of measured or calculated expectation functions versus the detector positions.

For applying the maximum-likelihood principle, it is mandatory to digitize each PM signal, to select a significant pair, and to 
TABLE I

COMPARISON OF Properties of BOth DATA HANDLING METHOdS

\begin{tabular}{l|l|l}
\hline Properties & Maximum-likelihood Principle & Center of Mass Principle \\
\hline Linearity of detector response & Perfect & Moderate and poor at both ends \\
\hline Width of point spread functions & Somewhat position dependent & $\begin{array}{l}\text { Position dependent Always smaller } \\
\text { than than in the ML case }\end{array}$ \\
\hline Stability of detector signals & Highly required & Highly required \\
\hline Adjustment of detector signals & Not needed & $\begin{array}{l}\text { Necessary, otherwise additional } \\
\text { non-linearities }\end{array}$ \\
\hline $\begin{array}{l}\text { Influence of signal noise on } \\
\text { spatial resolution }\end{array}$ & Only from the two involved signals & From all signals \\
\hline Required information & $\begin{array}{l}\text { All signals SO, } \mathrm{S1}, \ldots . . \\
\text { All expectation functions } \mathrm{B}_{i}(\mathrm{x})\end{array}$ & All signals S0, S1,...only \\
\hline
\end{tabular}

determine the increment channel. For measurements of the expectation functions, which have to be done once, it is required to make digitized signals of all PMs available in a PC.

Since the digital data processing becomes state of the art the maximum-likelihood principle will get a chance of realization.

Presently in our laboratory, a huge two-dimensional scintillation detector for subthermal neutrons is under construction. This development implies two test facilities for measuring:1) the uniformity of the PMs and 2) the light distribution functions. These measured information are implemented in the computer codes for filling the tables. In addition, the light distribution calculations are refined to get close to a real scintillation detector. This subject will be described in a separate paper in which the two dimensional case of the maximum likelihood principle is treated.

\section{ACKNOWLEDGMENT}

The authors are grateful to K. Zwoll for valuable discussions and to S. Bippus for correcting and formatting the final version of the manuscript.

\section{REFERENCES}

[1] J. Schelten, R. Kurz, I. Naday, and W. Schäfer, "Development of a linear position-sensitive neutron detector," Nucl. Instrum. Methods Phys. Res. vol. A 205, pp. 319-330, 1983.

[2] M. Heiderich, R. Reinartz, R. Kurz, and J. Schelten, "A two-dimensional scintillation detector for small angle neutron scattering," Nucl. Instrum. Methods Phys. Res., vol. A 305, pp. 423-432, 1991.

[3] R. Kurz, R. Reinartz, S. Widdau, J. Schelten, A. Scholz, and W. Schäfer, "Two dimensional neutron detector based on a position sensitive photomultiplier," Nucl. Instrum. Methods Phys. Res., vol. A 273, pp. 273-282, 1988
[4] A. J. Bird, Z. He, and D. Ramsden, "Multi-channel readout of crossed-wire anode photomultipliers," Nucl. Instrum. Methods Phys. Res., vol. A 348, pp. 668-672, 1994.

[5] J. Schelten, R. Reinartz, R. Engels, M. Balzhäuser, J. Lauter, W. Schäfer, and K. D. Müller, "Position sensitive neutron detection with ${ }^{6} \mathrm{LiF}$ layers on silicon semiconductors," Nucl. Instrum. Methods Phys. Res., vol. A 389, pp. 447-453, 1997.

[6] A. Oed, "Position-sensitive detector with microstrip anode for electron multiplication with gases," Nucl. Instrum. Methods Phys. Res., vol. A 263, pp. 351-359, 1988.

[7] B. Gebauer, C. Schulz, and T. Wilpert, "Novel large area thermal neutron imaging detectors comprising ${ }^{157} \mathrm{Gd} / \mathrm{CsCl}$-convertors and micro-strip gas detectors with low-pressure, two-stage amplification and delay line readout," Nucl. Instrum. Methods Phys. Res., vol. A 392, pp. 68-72, 1997.

[8] E. Tanaka, T. Hiramo, and N. Nohara, "Scintillation cameras based on new postion arithmetics," J. Nucl. Med., vol. 11, pp. 542-547, 1970.

[9] T. Hiromoto, E. Tanaka, and N. Nohara, "A scintillation camera based on delay-line time conversion," J. Nucl. Med., vol. 12, pp. 160-165, 1971.

[10] N. Nohara, E. Tanaka, and T. Hiromoto, "High-resolution scinticamera based on delay-line time conversion," J. Nucl. Med., vol. 12, pp. 635-636, 1971.

[11] R. M. Gray and A. Macovski, "Maximum a posteriori estimation of position in scintillation cameras," IEEE Trans. Nucl. Sci., vol. 23, pp. 849-852, 1976.

[12] C. A. Burnham, J. Bradshaw, D. Kaufman, D. A. Chesler, C. W. Stearns, and G. L. Brownell, "Design of a cylindrical shaped scintillation camera for position tomographs," IEEE Trans. Nucl. Sci., vol. 32, pp. 889-893, 1985.

[13] N. H. Clinthorne, W. L. Rogers, L. Shao, and K. F. Koral, "A hybrid maximum likelihood position computer for scintillation cameras," IEEE Trans. Nucl. Sci., vol. 34, pp. 97-101, 1987.

[14] X. Liu, N. H. Clinthorne, L. Shao, K. F. Koral, and W. L. Rogers, "Recursive calculation of the two-dimensional maximum likelihood position estimation for a scintillation camera," IEEE Trans. Nucl. Sci., vol. 37, pp. 713-717, 1987.

[15] T. D. Milster, J. A. Selberg, H. H. Barrett, A. L. Landesman, and R. H. Seacat III, "Digital position estimation for the modular scintillation camera," IEEE Trans. Nucl. Sci., vol. 32, pp. 748-752, 1985.

[16] T. D. Milster, J. A. Selberg, H. H. Barrett, R. L. Easton, G. R. Rossi, J. Arendt, and R. G. Simpson, "A modular scintillation camera for use in nuclear medicine," IEEE Trans. Nucl. Sci., vol. 31, pp. 578-580, 1984. 